\title{
Antigen-specific acquired immunity in human brucellosis: implications for diagnosis, prognosis, and vaccine development
}

\section{Anthony P. Cannella ${ }^{1}$, Renee M. Tsolis ${ }^{2}$, Li Liang ${ }^{3}$, Philip L. Felgner ${ }^{3}$, Mayuko Saito ${ }^{4}$, Alessandro Sette ${ }^{5}$, Eduardo Gotuzzo ${ }^{6}$ and Joseph M. Vinetz ${ }^{1 *}$}

\author{
1 Division of Infectious Diseases, Department of Medicine, University of California San Diego, La Jolla, CA, USA \\ ${ }^{2}$ Department of Medical Microbiology and Immunology, University of California Davis, Davis, CA, USA \\ ${ }^{3}$ Division of Infectious Diseases, Department of Medicine, University of California Irvine, Irvine, CA, USA \\ ${ }^{4}$ Asociacion Benefica PRISMA, Lima, Peru \\ ${ }^{5}$ Center for Infectious Diseases, Allergy and Asthma, La Jolla Institute of Allergy and Immunology, La Jolla CA, USA \\ ${ }^{6}$ Alexander von Humboldt Institute of Tropical Medicine, Universidad Peruana Cayetano Heredia, Lima, Peru
}

\section{Edited by:}

Thomas A. Ficht, Texas A\&M

University, USA

\section{Reviewed by:}

Yongqun "Oliver" He, University of Michigan School of Medicine, USA Menachem Banai, Kimron Veterinary Institute, Israel

\section{*Correspondence:}

Joseph M. Vinetz, Division of Infectious Diseases, Department of Medicine, University of California San Diego, Palade Laboratories, Room \#125, 9500 Gilman Drive, La Jolla, CA 92092-0741, USA. e-mail: jvinetz@ucsd.edu
Brucella spp., are Gram negative bacteria that cause disease by growing within monocyte/macrophage lineage cells. Clinical manifestations of brucellosis are immune mediated, not due to bacterial virulence factors. Acquired immunity to brucellosis has been studied through observations of naturally infected hosts (cattle, goats), mouse models (mice), and human infection. Even though Brucella spp. are known for producing mechanisms that evade the immune system, cell-mediated immune responses drive the clinical manifestations of human disease after exposure to Brucella species, as high antibody responses are not associated with protective immunity. The precise mechanisms by which cell-mediated immune responses confer protection or lead to disease manifestations remain undefined. Descriptive studies of immune responses in human brucellosis show that $\mathrm{TH}_{1}$ (interferon$\gamma$-producing $T$ cells) are associated with dominant immune responses, findings consistent with animal studies. Whether these $T$ cell responses are protective, or determine the different clinical responses associated with brucellosis is unknown, especially with regard to undulant fever manifestations, relapsing disease, or are associated with responses to distinct sets of Brucella spp. antigens are unknown. Few data regarding T cell responses in terms of specific recognition of Brucella spp. protein antigens and peptidic epitopes, either by $\mathrm{CD} 4+$ or $\mathrm{CD} 8+\mathrm{T}$ cells, have been identified in human brucellosis patients. Additionally because current attenuated Brucella vaccines used in animals cause human disease, there is a true need for a recombinant protein subunit vaccine for human brucellosis, as well as for improved diagnostics in terms of prognosis and identification of unusual forms of brucellosis. This review will focus on current understandings of antigen-specific immune responses induced Brucella peptidic epitopes that has promise for yielding new insights into vaccine and diagnostics development, and for understanding pathogenetic mechanisms of human brucellosis.

Keywords: T cell epitope, immunology, Brucella, zoonotic diseases, systems biology

\section{INTRODUCTION}

Cellular mediated immune responses to brucellosis drive a broad range of manifestations of the disease that vary from subclinical infection (more common with Brucella abortus) to undulant fever, to focal pyogenic infection, to chronic fatigue syndromelike illness (Yingst and Hoover, 2003; Yang et al., 2005). However, the molecular mechanisms that determine the variable manifestations of Brucella infection remain to be elucidated. Information on acquired immunity to human brucellosis has been accumulated through observational studies of naturally infected hosts (cattle, goats), experimental models (mice), and observations of human disease. Three predominant Brucella species are seen frequently in human infections: B. melitensis, B. abortus, and B. suis. Of these three species, B. melitensis infections are most commonly seen in humans and seem to be the most pathogenic (Pappas et al., 2005). In the United States, domesticated cattle, which are potential reservoirs for the organism, are vaccinated against $B$. abortus (RB51 or S19); elsewhere (in the Middle East and Latin America), goats and sheep may be vaccinated with Rev-1, an attenuated strain of B. melitensis. Currently available veterinary vaccines are comprised of live-attenuated organisms but are unacceptable in humans because they cause clinical disease (Kinikli et al., 2005; Durward et al., 2010).

Brucellosis can occur in several forms: acute/subacute (associated with positive blood cultures, high titer agglutination serologies), focal (blood culture negative, serologically variable, and 
positive local site culture), and chronic (blood culture negative, serologically variable but often negative, sometimes bone marrow culture positive, often diagnosed clinically in response to therapy; Jimenez de Bagues et al., 2005). However, it is currently unknown what causes some individuals to have the acute form and some to progress and develop chronic disease. As hypothesized in this review and elsewhere, it seems probable that immunogenetics of cell-mediated immune responses to Brucella protein antigens determines clinical manifestations and outcome. Sometimes, despite treatment for brucellosis, there are still some bacterial foci that may persist despite antibiotics or Brucella DNAemia may persist, presumably because of deficient $\mathrm{T}$ cell activation of infected macrophage/dendritic cells (DCs; Vrioni et al., 2008). Additional mechanisms may also include altered innate immune responses determined by the pathogenetic properties of the bacteria themselves. There have been studies demonstrating that Brucella epitopes can include those recognized by peptide-specific CD8+ T cells associated with protective responses at least in a mouse model (Durward et al., 2010). While there has been an experimental interferon-gamma release assay developed for bovine brucellosis, there has not been one developed for human infection to differentiate immune responses associated with different forms of brucellosis, to definitely diagnose previous exposure, or identify targets of protective immunity. Understanding the precise molecular targets (protein, peptidic epitope) of T cell-mediated immune responses has the promise to translate to further investigations into new vaccine and diagnostic Brucella T cell epitopes, and their role in specific $\mathrm{T}$ cell-mediated responses.

\section{T CELL CYTOKINE RESPONSES TO BRUCELLA INFECTION}

Infections may occur after ingestion or inhalation of Brucellae that penetrate mucosal surface such as the upper respiratory or gastrointestinal mucosa via lymphoid cells. Once the bacteria are phagocytosed by macrophages, DCs (Billard et al., 2007), and other antigen presenting cells (APCs), approximately $40-50 \%$ of the bacteria resist digestion within these cells. B. abortus and B. melitensis that have smooth LPS (with intact $\mathrm{O}$-antigen chain) are able to survive better intracellularly than $B$. canis that has rough LPS (lacks O-antigen side chain; Vrioni et al., 2008). Brucella spp. LPS is also composed of longer carbon chains (C28) as apposed to the usual 12-16 carbons in the LPS from Enterobacteriaceae. In addition, Brucella spp. produce proteins (e.g., Vi antigen), which create a capsule around the LPS, therefore limiting it to have contact with TLR4 receptors (Lapaque et al., 2005; Tsolis et al., 2008). In addition, the domain for the flagellin protein in Brucella spp. does not stimulate TLR5 receptors, and is another way for the bacteria to evade the immune system during early infection (Tsolis et al., 2008). These areas are conserved in all Brucella spp. and to others in the same family to evade detection by the immune system during the infection and possibly allowing the bacteria to persist in the reticuloendothelial system (Tsolis et al., 2008; Barquero-Calvo et al., 2009).

After initial encounter with Brucella antigens, APCs produce interleukin-1 (IL-1), interleukin-6 (IL-6), tumor necrosis factor alpha (TNF- $\alpha$ ), and gamma-interferon (IFN- $\gamma$ ) initiating innate immune responses (including natural killer cells) that may limit the initial spread of organisms. Infected APC in which organisms residing within unactivated phagolysosomes are likely to present some subset of peptidic Brucella antigens (hitherto unknown) to $\mathrm{CD} 4+$ and $\mathrm{CD} 8+$ cells, and thus inducing a $\mathrm{TH}_{1}$ response associated with IFN- $\gamma$ release. The functional consequences of antigen-specific IFN- $\gamma$ release is unclear but does not lead to elimination of organisms during active, symptomatic infection and likely results in clinical symptomatology (i.e., fever, sweating, weight loss). Clonal $\mathrm{T}$ cell expansion is initiated with production of interleukin-2 (IL-2) and interleukin-12 (IL-12), which initiates a CD8+ cytotoxic response on Brucella-infected cells. Infected macrophages produce IL-12 and IFN- $\gamma$ which regulate antigen presentation and may contribute to the limitation of intracellular bacterial replication through unknown mechanisms (Akbulut et al., 2005). Data also suggest that Brucella spp. also modify the initial immune response once phagocytosed into APCs. To survive within APCs, Brucella use certain gene products to subvert certain phagocyte intracellular processes, particularly phagosomelysosome fusion which would be associated with bacterial killing. One way that the organism survives within APCs is through Brucella-containing vacuoles (BCVs), which the organism secretes Sar1, a critical protein which allows the organism to replicate within these vacuoles (Celli et al., 2005). CD64, also known as $\mathrm{Fc} \gamma \mathrm{RI}$, is a macrophage-expressed gene whose expression is down-regulated in B. melitensis infections (Lapaque et al., 2005), reflecting the reduction of antigen processing in Brucella-infected APCs and possibly inhibiting the killing of infected cells initiated by $\mathrm{CD} 4+$ and CD8+ T cells. This mechanism may be important for decreasing superoxide and reactive nitrogen compounds as another pathogen-associated localized immunosuppression. Other gene products such as cystatin C, serpina3c, and Gas2 (natural peptidase inhibitors) appear to alter macrophage chemotaxis, cell migration, and proliferation, which may further allow Brucella to avoid immune surveillance and lead to enhanced multiplication (Lapaque et al., 2005). Decreased transcription of Cyp4a10 is thought to be associated with the reduction of oxidative stress that creates an environment conducive to bacterial proliferation. Prkca is another macrophage-associated gene potentially modulated during Brucella infection, who functions includes regulating phagosome-lysosome fusion and intracellular vesicle and reactive oxygen species (ROS) generation. Prkca expression is dramatically reduced in Brucella-infected macrophages, and interestingly, has been shown to be regulated by other intracellular pathogens such as Salmonella, Leishmania, and Legionella. All these mechanisms contribute to allowing Brucellae to survive within the intracellular environment, and evade not only the innate immunity, but CD4+- and CD8+-mediated host cell killing (Covert et al., 2009).

Alteration of $\mathrm{T}$ cell function may be key to explaining the clinico-pathological manifestations of chronic or relapsing brucellosis. Specifically, a decreased $\mathrm{TH}_{1}$ cytokine response by APCs (with decreased activation of cytotoxic T cells via IFN- $\gamma$, IL-12, and possibly IL-17 (Pasquevich et al., 2010), and toward a $\mathrm{TH}_{2}$ response (which decreases phagocyte function and reduced cytotoxic response via IL-4, IL-5, and IL-10; Jimenez de Bagues et al., 2005). These infected cells fail to produce IFN- $\gamma$, and decrease clonal expansion of Brucella-specific CD4+ T cells. Subsequently, Brucella-specific CD8 $+\mathrm{T}$ cells would not initiate destruction 
of infected cells by perforin and granzyme injection (cell lysis) or through stimulation of FAS ligand (cellular apoptosis; Yingst and Hoover, 2003; Skendros et al., 2008). Based on these considerations, the unusual and diverse manifestations of chronic and relapsing brucellosis could be related to several potential immunopathogenic mechanisms: an ineffective CD4+ effector response, a down-regulated $\mathrm{CD} 8+\mathrm{T}$ cell response or a continued, established $\mathrm{TH}_{2}$ response, each of which could result in an incomplete resolution of the infection (Giambartolomei et al., 2004). In comparing cytokine responses in patients with acute and chronic brucellosis, before and after treatment, Akbulut et al. (2005) found that in chronic brucellosis, both the absolute number of CD4+ cells and the quantitative secretion of IFN- $\gamma$ were reduced. Rafiei et al. (2006) confirmed these results, and further demonstrated that IL-13 is increased in chronic brucellosis, further demonstrating the initial association of $\mathrm{TH}_{1}$ cytokine responses with acute brucellosis illness, which gradually becomes $\mathrm{TH}_{2}$ cytokine dominant. These observations suggest that once acute brucellosis has resolved, that both the number of CD4+ cells and CD4+ functional response is reduced. Whether antigen-specific memory CD4+ cells are produced during acute or chronic brucellosis remains to be determined (Moreno-Lafont et al., 2002; Akbulut et al., 2005; Kinikli et al., 2005). Recently Elfaki and Al-Hokail observed that mice deficient in $\beta 2$-microglobulin produced an impaired CD8+ response associated with increased Brucella bacterial load and decreased clearance (Moreno-Lafont et al., 2002; Elfaki and Al-Hokail, 2009). Extrapolating such results to human disease, chronic or relapsing brucellosis might be explained in that CD69 expression on both CD4+ T cells and CD8+ T cells are significantly decreased in this patient subset. Further experimentation indicates that there is an unknown individual effect on the immune system after an episode of acute brucellosis, and therefore one develop chronic and relapsing brucellosis.

\section{BRUCELLA PROTEINS RECOGNIZED IN HUMAN IMMUNE RESPONSES}

Epitopes are the molecular subset of any macromolecule recognized by antibodies, B cells, T cells, and NK cells. T cells recognize peptides generally of 8-20 amino acids bound to MHC molecules (HLA Class I associated with CD8+ T cells; HLA Class II associated with CD4+ T cells) presented on the surface of a B cell, macrophage or dendritic cell (Yang et al., 2005). Non-classical MHC molecules also present non-peptidic epitopes such as glycolipids to non-restricted lymphocytes, particularly NK cells. There are only a few studies in the literature that have demonstrated either a humoral or cellular response to Brucella epitopes. The epitopes of greatest interest to date include Bp26 (a periplasmic protein), Trigger Factor (a chaperone protein; Yang et al., 2005), and the outer membrane lipoproteins such as Omp 10, 16, and 19 (Tibor et al., 1999). Interestingly, these antigens, especially the outer membrane lipoproteins, appear to be potent in inducing cytokine responses from memory $\mathrm{T}$ cells. In contrast, Brucella LPS and Brucella DNA do not elicit intense immune responses (Giambartolomei et al., 2004; Vrioni et al., 2008). Previous vaccine studies in mice have used some of these outer membrane epitopes (Pasquevich et al., 2010) as well as certain enzymes such as $\mathrm{Cu}-\mathrm{Zn}$ superoxide dismutase (Singha et al., 2008). Yet, these were not demonstrated to offer protection for humans or $\mathrm{T}$ cell cytokine release assays to prove their efficacy.

These antigens have been identified in both B. melitensis and B. abortus. Bp26 and Trigger Factor have been shown to be recognized by the immune system (Yang et al., 2005), yet with a reduced activation in patients with chronic/relapsing brucellosis. Recently, Liang et al. $(2010,2011)$ reported comprehensive systems biology analyses of human antibody responses in acute $B$. melitensis brucellosis in Peru. A collection of sera isolated from individuals from one of the following groups was used to probe large scale $B$. melitensis protein microarrays including a $\sim 1400$ proteins array and 3300 proteins array representing nearly the entire encoded proteome: Brucella blood culture positive, blood culture negative with positive Rose Bengal, blood culture negative with negative Rose Bengal, and two naïve groups (from both American and Peruvian individuals.) Sets of proteins that differentiated acutely infected from uninfected patient groups were identified that were recognized by patient IgG responses. These Brucella protein epitopes were then further separated by the patient groups which recognized them, as some were only recognized by culture positive or culture negative patients/Rose Bengal positive patients compared to naïve patients (Table 1), others cross react in both the culture positive patients and the naive patients (Table 2) and some antigens produced a positive response in the culture positive group but not the culture negative group/Rose Bengal positive group (Table 3). This broad spectrum of antibody responses demonstrates the differences between in the manner that these groups respond to Brucella protein epitopes. Interestingly, some of the epitopes mentioned previously (i.e., Bp 26) produced a strong antibody response by Peruvian brucellosis patients, who were culture positive or culture negative patients/Rose Bengal positive patients. These antibody responses could reflect the substantial differences, which these protein epitopes have on the immune system activation, and a large number of antigens recognized by brucellosis patients were identified which have yet to be studied or have limited understanding of their function.

There could be a potential difference in the antibody responses compared to cytokine release assays for these specific epitopes, which produced antibody responses. Based on unpublished data, Bp26, which gave a strong antibody response, does not give a strong $\mathrm{TH}_{1}$ response; VirB8 does generate a strong $\mathrm{TH}_{1}$ response, yet does not give a particularly strong antibody response. The use of an epitope database has previously been used for Mycobacteria tuberculosis, and other infections by estimating the immune responses to epitopes associated with a certain organism (Blythe et al., 2007). A complete cytokine release assay for the entire brucellar proteome has not been undertaken. Based on the fact that antibody production is not protective in chronic brucellosis, we must conclude that $\mathrm{T}$ cell assays would be a more appropriate method to pursue with regards to not only diagnostic purposes, but for development of a recombinant protein vaccine as well.

\section{CONCLUSION}

Brucella spp. are important intracellular human and animal pathogens associated with fascinating mechanisms of immune modulation and subversion of APCs as an intrinsic mechanism 
Table 1 | Serodiagnostic antigens for culture+ and culture-/Rose Bengal+ vs. Peruvian Naive.

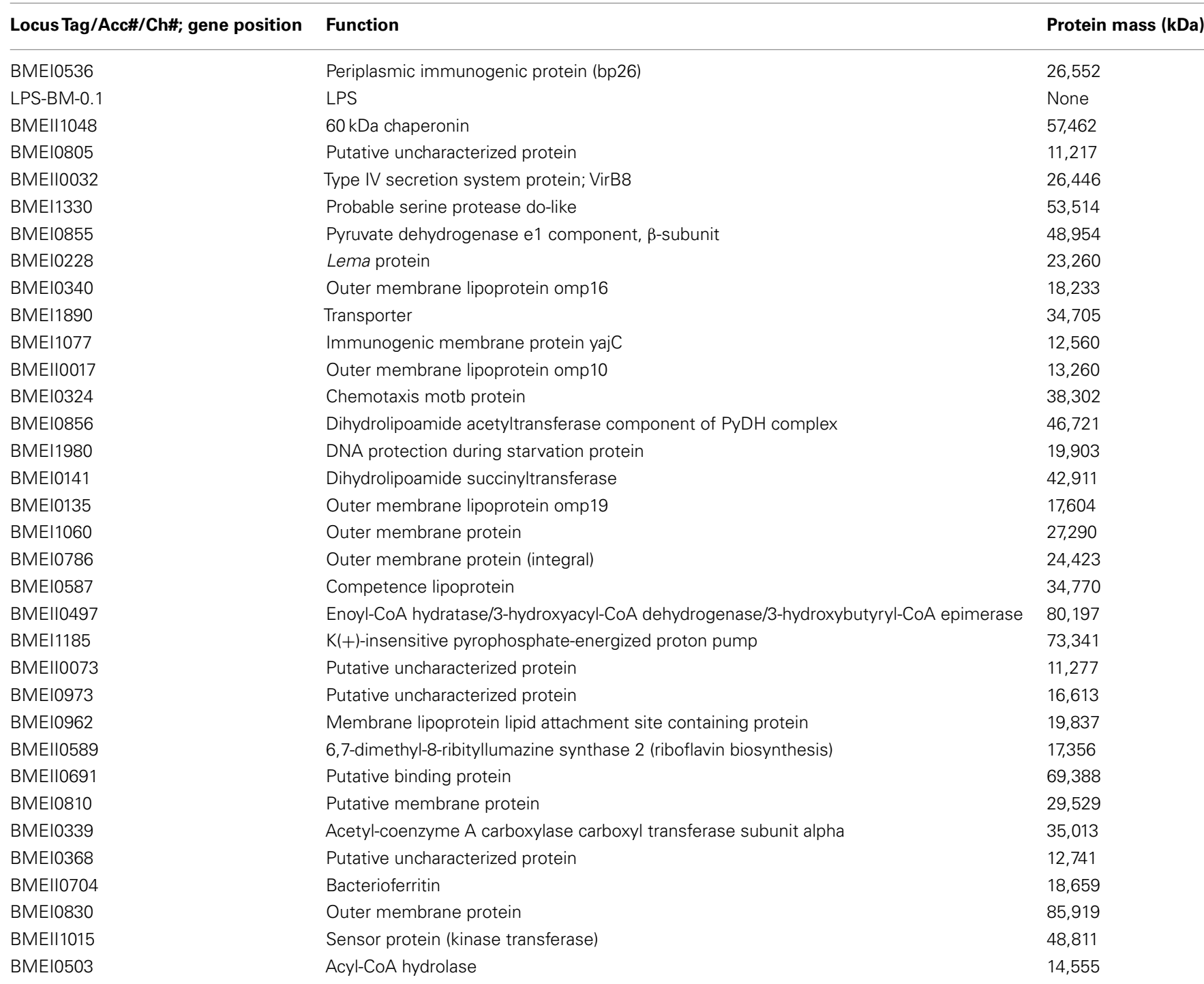

Table 2 | Cross-reactive antigens for culture+ or culture-/Rose Bengal+ vs. Peruvian Naïve (negative controls).

\begin{tabular}{lll}
\hline Locus Tag/Acc\#/Ch\#; gene position & Function & Protein mass (kDa) \\
\hline BMEI1079 & Putative outer membrane lipoprotein & 43,604 \\
BMEI1082 & Seryl-tRNA(Ser/Sec) synthetase & 47,520 \\
BMEI1084 & Sec-independent protein translocase protein tatA/E homolog & 7,999 \\
BMEII0010 & Hypothetical membrane associated protein & 67,136 \\
BMEl1033 & Putative uncharacterized protein & 23,107 \\
BMEII0917 & Putative uncharacterized protein & 17,923 \\
BMEII1111 & Putative uncharacterized protein & 16,629 \\
BMEII0040 & Glutamate synthase (large chain) & 173,600 \\
BMEII0356 & Galactonate dehydratase & 66,751 \\
BMEl1382 & Betaine aldehyde dehydrogenase & 52,035 \\
\hline
\end{tabular}

of the diseases that they cause. New systems biology analyses of antigens recognized by human immune responses in brucellosis have identified large numbers of protein antigens with potential for understanding mechanisms of pathogenesis and immune evasion and may point the way toward novel vaccine and diagnostic approaches. These approaches have generalized applicability to the 
Table 3 | Serodiagnostic antigens for culture+ vs. culture-/Rose Bengal+.

Locus Tag/Acc\#/Ch\#; gene position

BMEII0173
BMEII0029
BMEII0182
BMEII0054
BMEII1049
BMEII0558
BMEII0532
BMEII0892
BMEI1502
BMEII0720

BMEII0173

BMEII0029

BMEII0182

BMEII0054

BMEII1049

BMElI0558

BMEII0892

BMEII0720
Function

Protein mass (kDa)

Putative uncharacterized protein
Type IV secretion system protein virB5
Putative uncharacterized protein
Putative uncharacterized protein
Putative uncharacterized protein
Putative uncharacterized protein
Hypothetical membrane associated protein
Putative uncharacterized protein
Alkaline phosphatase like protein
Transcriptional regulator

11,277

26,810

8,718

5,237

7,955

7,596

7,916

6,762

21,488

7,304 analysis of $\mathrm{T}$ cell responses associated chronic bacterial, fungal and parasitic infections other than Brucella.

\section{ACKNOWLEDGMENTS}

This work was partially supported by grant K24AI068903, 3U01AI075420, AI087164, and T32AI007036 from the United

\section{REFERENCES}

Akbulut, H. H., Kilic, S. S., Bulut, V., and Ozden, M. (2005). Determination of intracellular cytokines produced by Th1 and Th2 cells using flow cytometry in patients with brucellosis. FEMS Immunol. Med. Microbiol. 45, 253-258.

Barquero-Calvo, E., Conde-Alvarez, R., Chacon-Diaz, C., Quesada-Lobo, L., Martirosyan, A., Guzmán-Verri, C., Iriarte, M., Mancek-Keber, M., Jerala, R., Gorvel, J. P., Moriyón, I., Moreno, E., and Chaves-Olarte, E. (2009). The differential interaction of Brucella and ochrobactrum with innate immunity reveals traits related to the evolution of stealthy pathogens. PLOS ONE 4, e5893. doi: 10.1371/journal.pone. 0005893

Billard, E., Dornand, J., and Gross, A. (2007). Interaction of Brucella suis and Brucella abortus rough strains with human dendritic cells. Infect. Immun. 75, 5916-5923.

Blythe, M. J., Zhang, Q., Vaughan, K., de Castro, R. Jr., Salimi, N., Bui, H. H., Lewinsohn, D. M., Ernst, J. D., Peters, B., and Sette, A. (2007). An analysis of the epitope knowledge related to Mycobacteria. Immunome Res. $3,10$.

Celli, J., Salcedo, S. P., and Gorvel, J. P. (2005). Brucella coopts the small GTPase Sarl for intracellular replication. Proc. Natl. Acad. Sci. U.S.A. 102, 1673-1678.

Covert, J., Mathison, A. J., Eskra, L., Banai, M., and Splitter, G. (2009).
Brucella melitensis, B. neotomae and B. ovis elicit common and distinctive macrophage defense transcriptional responses. Exp. Biol. Med. (Maywood). 234, 1450-1467.

Durward, M. A., Harms, J., Magnani, D. M., Eskra, L., and Splitter, G. A. (2010). Discordant Brucella melitensis antigens yield cognate CD8+ $\mathrm{T}$ cells in vivo. Infect. Immun. 78, 168-176.

Elfaki, M. G., and Al-Hokail, A. A. (2009). Transforming growth factor beta production correlates with depressed lymphocytes function in humans with chronic brucellosis. Microbes Infect. 11, 1089-1096.

Giambartolomei, G. H., Zwerdling, A., Cassataro, J., Bruno, L., Fossati, C. A., and Philipp, M. T. (2004). Lipoproteins, not lipopolysaccharide, are the key mediators of the proinflammatory response elicited by heat-killed Brucella abortus. J. Immunol. 173, 4635-4642.

Jimenez de Bagues, M. P., Dudal, S., Dornand, J., and Gross, A. (2005). Cellular bioterrorism: how Brucella corrupts macrophage physiology to promote invasion and proliferation. Clin. Immunol. 114, 227-238.

Kinikli, S., Turkcapar, N., Kucukay, M. B., Keskin, G., and Kinikli, G. (2005). In vitro nonspecific mitogenic response of T-cell subsets in acute and chronic brucellosis. Diagn. Microbiol. Infect. Dis. 52, 229-233.

Lapaque, N., Moriyon, I., Moreno, E., and Gorvel, J. P. (2005). Brucella

States Public Health Service, National Institutes of Health. We would like to thank Ms. Paula Maguina of UC San Diego, who made contributions to both research management and international collaboration. Finally, we would like to thank all of the patients both in the USA and in Peru for their participation in this study.

lipopolysaccharide acts as a virulence factor. Curr. Opin. Microbiol. $8,60-66$.

Liang, L., Leng, D., Burk, C., NakajimaSasaki, R., Kayala, M. A., Atluri, V. L., Pablo, J., Unal, B., Ficht, T. A., Gotuzzo, E., Saito, M., Morrow, W. J., Liang, X., Baldi, P., Gilman, R. H., Vinetz, J. M., Tsolis, R. M., and Felgner, P. L. (2010). Large scale immune profiling of infected humans and goats reveals differential recognition of Brucella melitensis antigens. PLoS Negl. Trop. Dis. 4 , e673.

Liang, L., Tan, X., Juarez, S., Villaverde, H., Pablo, J., Nakajima-Sasaki, R., Gotuzzo, E., Saito, M., Hermanson, G., Molina, D., Felgner, S., Morrow, W. J., Liang, X., Gilman, R. H., Davies, D. H., Tsolis, R. M., Vinetz, J. M., and Felgner, P. L. (2011). Systems biology approach predicts antibody signature associated with $\mathrm{Bru}$ cella melitensis infection in humans. J. Proteome Res. 10, 4813-4824.

Moreno-Lafont, M. C., Lopez-Santiago, R., Zumaran-Cuellar, E., ParedesCervantes, V., López-Merino, A., Estrada-Aguilera, A., and SantosArgumedo, L. (2002). Antigenspecific activation and proliferation of CD4+ and CD8+ T lymphocytes from brucellosis patients. Trans. $R$. Soc. Trop. Med. Hyg. 96, 340-347.

Pappas, G., Akritidis, N., Bosilkovski, M., and Tsianos, E. (2005). Brucellosis. N. Engl. J. Med. 352, 2325-2336.

Pasquevich, K. A., Garcia Samartino, C., Coria, L. M., Estein, S. M.,
Zwerdling, A., Ibañez, A. E., Barrionuevo, P., Oliveira, F. S., Carvalho, N. B., Borkowski, J., Oliveira, S. C., Warzecha, H., Giambartolomei, G. H., and Cassataro, J. (2010). The protein moiety of Brucella abortus outer membrane protein 16 is a new bacterial pathogen-associated molecular pattern that activates dendritic cells in vivo, induces a Th1 immune response, and is a promising self-adjuvanting vaccine against systemic and oral acquired brucellosis. J. Immunol. 184, 5200-5212.

Rafiei, A., Ardestani, S. K., Kariminia, A., Keyhani, A., Mohraz, M., Amirkhani, A. (2006). Dominant Thl cytokine production in early onset of human brucellosis followed by switching towards Th2 along prolongation of disease. J Infect. 53, 315-324.

Singha, H., Mallick, A. I., Jana, C., Isore, D. P., Goswami, T.K., Srivastava, S. K., Azevedo, V. A., Chaudhuri, P., and Owais, M. (2008). Escheriosomes entrapped DNA vaccine coexpressing $\mathrm{Cu}-\mathrm{Zn}$ superoxide dismutase and IL- 18 confers protection against Brucella abortus. Microbes Infect. 10, 1089-1096.

Skendros, P., Sarantopoulos, A., Tselios, K., and Boura, P. (2008). Chronic brucellosis patients retain low frequency of CD4+ T-lymphocytes expressing $\mathrm{CD} 25$ and $\mathrm{CD} 28$ after Escherichia coli LPS stimulation of PHA-cultured PBMCs. Clin. Dev. Immunol. 2008, 327346

Tibor, A., Decelle, B., and Letesson, J. J. (1999). Outer membrane proteins 
Omp10, Omp16, and Omp19 of Brucella spp. are lipoproteins. Infect. Immun. 67, 4960-4962.

Tsolis, R. M., Young, G. M., Solnick, J. V., and Baumler, A. J. (2008). From bench to bedside: stealth of enteroinvasive pathogens. Nat. Rev. Microbiol. 6, 883-892.

Vrioni, G., Pappas, G., Priavali, E., Gartzonika, C., and Levidiotou, S. (2008). An eternal microbe: Brucella DNA load persists for years after clinical cure. Clin. Infect. Dis. 46, e131-e136.
Yang, X., Hudson, M., Walters, N., Bargatze, R. F., and Pascual, D. W. (2005). Selection of protective epitopes for Brucella melitensis by DNA vaccination. Infect. Immun. 73, 7297-7303.

Yingst, S., and Hoover, D. L. (2003). T cell immunity to brucellosis. Crit. Rev. Microbiol. 29, 313-331.

Conflict of Interest Statement: The authors declare that the research was conducted in the absence of any commercial or financial relationships that could be construed as a potential conflict of interest.

Received: 10 October 2011; accepted: 11 January 2012; published online: 01 February 2012.

Citation: Cannella AP, Tsolis RM, Liang L, Felgner PL, Saito M, Sette A, Gotuzzo E and Vinetz JM (2012) Antigen-specific acquired immunity in human brucellosis: implications for diagnosis, prognosis, and vaccine development. Front. Cell. Inf. Microbio. 2:1. doi: 10.3389/fcimb.2012.00001

Copyright (c) 2012 Cannella, Tsolis, Liang, Felgner, Saito, Sette, Gotuzzo and Vinetz. This is an open-access article distributed under the terms of the Creative Commons Attribution Non Commercial License, which permits noncommercial use, distribution, and reproduction in other forums, provided the original authors and source are credited. 